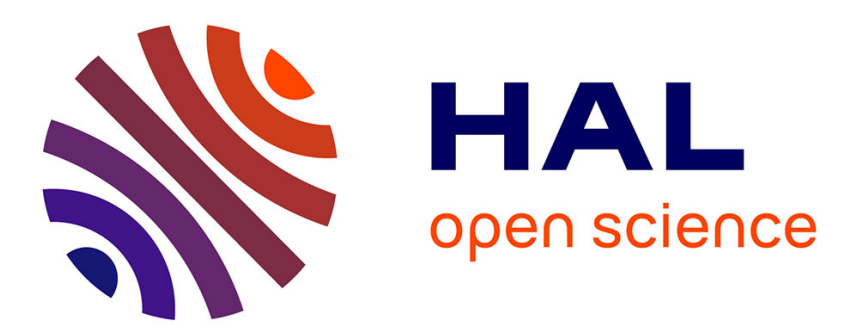

\title{
Discrimination à l'embauche selon l'origine et le genre : défiance indifférenciée ou ciblée sur certains groupes?
}

Anthony Edo, Nicolas Jacquemet

\section{To cite this version:}

Anthony Edo, Nicolas Jacquemet. Discrimination à l'embauche selon l'origine et le genre: défiance indifférenciée ou ciblée sur certains groupes?. Economie et Statistique / Economics and Statistics, 2014, 464-465-466, pp.155-172. halshs-00935241

\section{HAL Id: halshs-00935241 https://shs.hal.science/halshs-00935241}

Submitted on 11 Apr 2014

HAL is a multi-disciplinary open access archive for the deposit and dissemination of scientific research documents, whether they are published or not. The documents may come from teaching and research institutions in France or abroad, or from public or private research centers.
L'archive ouverte pluridisciplinaire HAL, est destinée au dépôt et à la diffusion de documents scientifiques de niveau recherche, publiés ou non, émanant des établissements d'enseignement et de recherche français ou étrangers, des laboratoires publics ou privés. 


\title{
Discrimination à l'embauche selon l'origine et le genre : défiance indifférenciée ou ciblée sur certains groupes?
}

\author{
Anthony Edo, Nicolas Jacquemet ${ }^{*}$
}

L'existence d'une discrimination raciale à l'embauche sur le marché du travail est désormais largement documentée dans la plupart des économies occidentales. Si ce phénomène est confirmé par différentes études pour le marché du travail français, les causes et les sources de cette discrimination restent quant à elles largement inconnues. Cet article présente les résultats d'une étude destinée à évaluer empiriquement les principales sources de discrimination à l'embauche en Île-de-France, en s'inspirant des développements récents de la littérature. Cette évaluation repose sur un envoi contrôlé de candidatures en réponse à des offres d'emploi postées sur des sites publics d'information.

L'étude montre que la discrimination à l'embauche à l'encontre des candidats issus de l'immigration est de l'ordre de $40 \%$ en moyenne. Cette inégalité de traitement entre candidats affecte toutes les candidatures d'origine étrangère, indépendamment de l'origine du candidat, ce qui va à l'encontre de l'hypothèse d'une défiance ciblée à l'encontre de vagues particulières d'immigration. Sur la base de ces résultats, il semble donc que la question de la discrimination se pose bien plus en termes d'opposition entre le groupe majoritaire, " autochtone », et l'ensemble des individus issus de l'immigration, qu'en termes de difficultés spécifiques propres à certains groupes. En ce sens, les résultats confirment l'existence d'une « homéophilie » ethnique sous-jacente à la discrimination - c'est à dire d'une défiance indifférenciée de la part des employeurs à l'égard de tout candidat n'appartenant pas au groupe ethnique majoritaire. Ensuite, nos résultats indiquent que les candidatures féminines sont favorisées par rapport à leurs équivalents masculins et relativement moins affectées par la discrimination d'origine. Enfin, l'inclusion d'un signal explicite d'aisance linguistique sur la moitié des envois élimine toute discrimination liée à l'origine pour les candidatures féminines. L'effet d'un tel signal est en revanche plus faible sur la discrimination opérant entre candidatures masculines.

La discrimination à l'égard des minorités ethniques est un facteur déterminant de l'émergence des inégalités liées aux origines dans l'accès à l'emploi. Sur la base de nombreux travaux empiriques de terrain, l'existence d'un comportement discriminatoire des employeurs à l'égard des minorités visibles est désormais largement admise - cf. Bertrand et Mullainathan

\footnotetext{
*Ecole d'Economie de Paris et Université Paris I Panthéon-Sorbonne. MSE, 106 Boulevard de l'hôpital, 75013 Paris France. anthony.edo@univ-paris1.fr ; nicolas.jacquemet@univ-paris1.fr. Nous remercions Clémence Berson, Eric Cédiey, Yannick 1'Horty, Amine Ouazad, Marie-Anne Valfort et Constantine Yannelis pour leurs remarques précieuses à différentes étapes de ce travail. Nous tenons également à remercier le CEPREMAP pour son soutien financier.
} 
(2005), Oreopoulos (2011), Oreopoulos et Dechie (2012), ainsi que Riach et Rich (2002) et Lang et Lehmann (2012) pour des revues de la littérature. Malgré une grande diversité en termes de zone géographique (Amérique du Nord, Europe du Nord) comme d'origine testée (immigrants asiatiques, indiens, d'Afrique du nord, etc.), ces travaux concluent en général à un handicap de l'ordre de $40 \%$ à $60 \%$ pour les candidats issus de l'immigration, et ce en raison de leur seule origine. Ces résultats ont été confirmés par quelques études appliquées au marché du travail français. Duguet \& al. (2010) observent par exemple un taux de discrimination de $35 \%$ à l'encontre des candidats d'origine maghrébine.

Deux fondements théoriques sont classiquement avancés pour comprendre la pratique des comportements discriminatoires. Le premier s'appuie sur les préférences des employeurs, le second met en évidence un calcul de leur part destiné à pallier les défauts d'information. Introduit par Becker (1957), le premier mécanisme est fondé sur l'existence d'un désagrément pour les employeurs à collaborer avec certaines minorités ethniques. Selon cette explication, les employeurs qui pratiquent la discrimination traitent ainsi de façon défavorable les travailleurs issus de minorités ethniques même si leurs caractéristiques productives sont identiques à celles du reste de la population. Le second mécanisme (Arrow, 1973 ; Phelps, 1972), repose sur l'idée que les employeurs n'évaluent qu'imparfaitement les caractéristiques productives d'un candidat à l'embauche. Pour les évaluer, ils se fondent alors non seulement sur les caractéristiques observables du candidat, mais également sur l'information contenue dans la distribution des caractéristiques productives au sein de son groupe démographique d'appartenance. En ce sens, cette forme de discrimination relève d'une pratique purement statistique de la part de l'employeur. Par conséquent, la discrimination à l'embauche est de nature statistique si deux candidats de productivité identique sont traités de façon inégale sur la seule base des performances moyennes, réelles ou supposées, du groupe auquel ils appartiennent (cf. Coate et Loury, 1993, pour une étude complémentaire sur ce sujet).

Par ailleurs, la littérature récente en économie (Currarini et al., 2009 ; Miguel et Gugerty, 2005) comme en sciences politiques (Putnam, 2007) montre que la distance ethnique en tant que telle constitue un facteur structurant des relations entre individus originaires de groupes ethniques différents. Sur la base de jeux expérimentaux classiques de mesure des préférences sociales, Habyarimana et al. (2009) montrent par exemple que la coopération au sein d'une communauté est d'autant plus faible que le degré de diversité ethnique qui la caractérise est important. Ils montrent en outre que cette différence ne peut être reliée à des variations de préférences, au sens où les comportements en termes d'altruisme membres apparaissent comme indifférenciés selon l'origine ethnique du partenaire. ${ }^{1}$ Les différences de comportement à l'égard d'autres groupes ethniques

1 Le degré de coopération au sein d'un groupe est mesuré par le niveau des contributions individuelles à un bien public (jeu dans lequel chaque membre du groupe choisit de répartir la somme qui lui est confiée entre un bien privé et un bien public, dont la rentabilité individuelle est plus faible mais qui profite à tous les membres du groupe). Le degré d'altruisme est mesuré à partir d'un jeu du dictateur, dans lequel une personne doit librement décider du partage entre elle-même et son partenaire de la somme d'argent qui lui est attribuée. Ces expériences ont été menées dans une localité d'Ouganda, caractérisée par une forte diversité ethnique. 
apparaissent d'après ces résultats comme le reflet d'une plus grande difficulté à identifier les comportements attendus, les normes et les croyances, en présence d'individus issus de groupes ethniques différents - quels qu'ils soient. Les individus issus d'un même groupe ethnique partageraient donc des caractéristiques communes qui facilitent la communication et favorisent le développement d'une confiance mutuelle. ${ }^{2}$

Appliqués au marché du travail, ces résultats suggèrent un nouvel axe d'analyse des comportements discriminatoires, compris comme le traitement particulier qu'un individu réserve aux membres de son propre groupe - par opposition à la vision traditionnelle d'une défiance ciblée à l'égard de groupes ou de minorités particuliers. Une telle propension d'un individu à privilégier les membres de son groupe d'appartenance est appelée " homéophilie » ethnique dans la suite de cet $\operatorname{article}^{3}$. Il faut noter que la notion d'homéophilie ethnique ne se présente pas comme une alternative, mais bien comme un raffinement des deux mécanismes traditionnels présentés plus haut. Ce type de comportement peut en effet être justifié tant par une préférence pour les membres de son propre groupe que par un calcul statistique opposant les individus selon qu'ils appartiennent ou non à ce groupe. La principale conséquence de l'homéophilie est qu'elle conduit à appliquer le même traitement - éventuellement discriminatoire - à tout individu issu d'un groupe différent. Pour tester cette hypothèse, Jacquemet et Yannelis (2011) ont évalué aux Etats-Unis le succès sur le marché du travail de candidats dont les noms sont sans ambigüité à consonance étrangère, mais dont l'origine ethnique est inconnue - i.e. non identifiée - des employeurs. Les résultats de l'expérience qu'ils mènent à Chicago montrent que le taux de discrimination à l'encontre de ces candidatures particulières est identique à celui que subissent les candidatures à consonance afro-américaine, confirmant l'hypothèse d'une homéophilie ethnique sous-jacente à la discrimination généralement observée.

Cet article présente le protocole et les résultats préliminaires d'une expérience destinée à tester ce mécanisme sur le marché du travail français. Nous répliquons pour ce faire l'étude de Jacquemet et Yannelis (2011) en créant trois profils d'individus qui ne diffèrent que par l'origine perçue induite par leur nom de famille, dont la consonance est soit française, soit maghrébine, soit étrangère mais inconnue de la population. La comparaison des taux de succès des candidats ainsi créés permet de mesurer non seulement le handicap que subissent les minorités ethniques dont l'origine est clairement identifiée par les employeurs, mais aussi

\footnotetext{
${ }^{2}$ Ces études se concentrent sur les modalités de coordination à l'intérieur d'un groupe caractérisé par une certaine diversité. Cet aspect est loin cependant d'épuiser l'ensemble des dimensions du lien entre la performance d'un groupe et sa diversité ethnique. Ottaviano et Peri (2006) montrent par exemple un lien de causalité positif entre le degré de diversité au sein des villes américaines (mesuré, entre autres, par la part des immigrés dans la population totale) et la productivité des natifs. Alesina et La Ferrara (2005) proposent une revue exhaustive de la littérature dans laquelle sont détaillés les effets positifs et négatifs potentiels induit par le degré de diversité sur la performance économique des pays.

3 Le néologisme « homéophilie » que nous introduisons désigne au sens étymologique l'attirance pour le semblable.
} 
la discrimination à l'encontre de candidats dont la seule spécificité est d'être issus de l'immigration, sans autre identification de leur origine.

A cet objectif s'ajoute la volonté de dresser un état des lieux aussi complet que possible des déterminants et de l'ampleur de la discrimination d'origine à l'embauche sur le marché du travail français. A cet effet, nous nous inspirons d'abord des travaux de Dechie et Oreopolous (2012) au Canada afin d'étudier la dimension «statistique » de la discrimination. Nous nous intéressons en particulier à l'effet des croyances (réelles ou supposées) des employeurs selon lesquelles les minorités ethniques auraient une moins bonne maîtrise de la langue française (qualité rédactionnelle et orale, aisance en grammaire et orthographe, richesse du vocabulaire, etc.). Pour tester empiriquement cette source de discrimination, nous introduisons une variable de traitement supplémentaire à travers un signal attestant d'une « bonne » maîtrise du langage pour les différents candidats. Cette modification des croyances des employeurs nous permettra de mesurer la variation du degré de discrimination en fonction de la présence d'un tel signal.

Ensuite, nous approfondissons les travaux de Duguet et Petit (2005) quant au rôle des discriminations liées au genre en créant une candidature féminine pour chacun des trois groupes de candidats. Nous sommes ainsi en mesure d'évaluer l'effet du genre à la fois sur le succès des candidatures conditionnellement à l'origine, et sur le taux de discrimination à l'égard des populations issues de l'immigration. ${ }^{4}$

Enfin, l'étude est conçue de manière à pouvoir prendre en compte le lien entre les pratiques discriminatoires et certaines caractéristiques de l'employeur. Afin d'évaluer l'effet de la discrimination à l'embauche sur la ségrégation spatiale, nous tenons compte de la localisation géographique de l'employeur. D'autre part, nous proposons d'utiliser les noms et prénoms des recruteurs indiqués sur les offres d'emploi auxquelles nous avons répondu pour créer un indicateur de proximité (en termes de genre) entre le recruteur et le candidat.

Nous résumons ci-dessous les principaux résultats connus quant à la discrimination d'origine et de genre sur le marché du travail français. Nous détaillons ensuite les différentes dimensions de l'étude : le choix de l'identité des candidats, les caractéristiques objectives ajoutées au $\mathrm{CV}$, puis les conditions pratiques de réalisation de l'envoi contrôlé des candidatures ainsi construites. Nous présentons ensuite les résultats issus de l'évaluation.

\section{Les évaluations par correspondance révèlent l'existence d'une discrimination à l'embauche en France...}

\footnotetext{
${ }^{4}$ Le croisement des différents motifs de discrimination, et l'analyse de leurs effets conjoints, restent peu étudiés en économie. Ils ont en revanche suscité une littérature importante en sociologie, et notamment en sociologie féministe depuis les travaux fondateurs de Crenshaw (1991).
} 
L'approche empirique privilégiée pour mesurer le taux de discrimination qui sépare deux candidatures identiques à l'exception de l'origine perçue du candidat est la méthode d'évaluation par correspondance, développée dans les années 1970 et 1980 au royaume Uni (Jowell et Prescott-Clarke, 1970 ; Firth, 1981, 1982). Cette technique a ensuite été popularisée par son application aux États-Unis par Bertrand et Mullainathan (2005), puis utilisée dans divers pays. La mesure de la discrimination repose sur un envoi contrôlé de candidatures fictives en réponses à des offres d'emploi réelles. Ces envois utilisent des CV cohérents avec les compétences demandées et sur lesquels apparaissent les noms des candidats. Les noms sont choisis de manière à indiquer aussi clairement que possible l'origine du candidat. Les noms jouent ainsi le rôle de variable de traitement, permettant de mesurer la discrimination, puisque les envois multiples de $\mathrm{CV}$ permettent de considérer que seule l'origine suggérée par le nom du candidat distingue les CV aux yeux des recruteurs.

La première application de l'évaluation par correspondance au marché du travail français date à notre connaissance de 2002 et s'intéresse aux discriminations de genre dans le

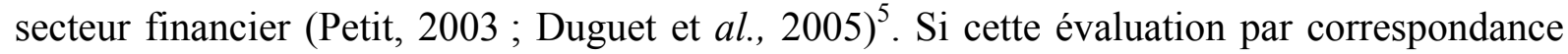
confirme que les femmes jeunes et sans enfant subissent un handicap important dans l'accès à un entretien d'embauche par rapport à leurs équivalents masculins, elle montre également que ce handicap disparaît complètement pour les candidat(e)s plus âgé(e)s. Depuis cette première étude, l'essentiel de l'attention s'est portée sur la discrimination liée à l'origine ethnique. L'une des toutes premières études date de 2006 et teste de façon simultanée les effets de la commune de résidence (favorisée ou non), de la nationalité et de l'origine du nom de famille et du prénom (française ou marocaine) sur les chances d'être invité à un entretien d'embauche dans le secteur de la comptabilité (Duguet et al., 2010). Les résultats confirment sans ambigüité l'existence d'une forte discrimination à l'encontre des candidats dont le nom et le prénom sont d'origine maghrébine. Pour recevoir une invitation à un entretien d'embauche, un candidat ayant un nom et un prénom français doit ainsi envoyer $19 \mathrm{CV}$ en moyenne alors qu'un candidat d'origine maghrébine doit en envoyer 54. L'évaluation par correspondance de Berson (2012) prolonge ces dernières études en appréhendant la relation entre le degré de concurrence dans le secteur de la distribution et le niveau de discrimination à l'embauche. Si les résultats indiquent qu'un fort degré de concurrence au sein d'un bassin d'emploi particulier suffit à faire disparaître la discrimination de genre qui globalement opère en faveur des femmes, le niveau de discrimination à l'encontre de candidats marocains lui est insensible.

Aeberhardt et al. (2010) ont par la suite étendu l'évaluation des discriminations ethniques sur le marché du travail français aux différentiels de salaire liés à l'origine des employés. Ils montrent que si les employés d'origine africaine subissent effectivement une

\footnotetext{
${ }^{5}$ Les premières études statistiques de la discrimination en France ont été menées sous l'égide du Centre de Recherche et d'Etudes des Dysfonctions de l'Adaptation (Bovenkerk et al. 1979) puis du BIT (Cédiey et Foroni, 2007). Ces études s'appuient sur des méthodes différentes de l'évaluation par correspondance, qui utilisent notamment les caractéristiques physiques des candidats comme signal de leur origine.
} 
pénalité salariale en raison de leur origine, l'essentiel des discriminations passe par le processus de recrutement plutôt que par la fixation des salaires. Enfin, Berson (2009) confirme que les différentiels de salaire liés aux origines sont transversaux aux secteurs d'activité, et apparaissent tant dans le secteur public que privé.

Si ces travaux convergent sans ambigüité vers l'existence d'une discrimination sur le marché du travail français à l'encontre des employés issus de l'immigration, les causes et les sources de ces discriminations restent largement inexplorées. Afin de combler cette lacune, l'étude présentée dans cet article se propose d'appliquer au cas français un certain nombre des développements récents de la littérature empirique consacrée à cette question.

\section{...qui pourraient provenir : des caractéristiques perçues des postulants induites par leur identité (variables de traitement I)...}

L'objectif premier de cette étude est de tester et évaluer empiriquement le rôle de ce qu'on appel ici l'homéophilie ethnique dans la discrimination à l'embauche généralement observée - i.e. l'existence d'une défiance indifférenciée de la part des employeurs à l'égard de tout candidat n'appartenant pas à son groupe ethnique, plutôt qu'une seule défiance à l'encontre de minorités ethniques clairement identifiées. Pour ce faire nous adaptons au cas français l'approche empirique développée par Jacquemet et Yannelis (2011).

La première étape consiste à construire trois couples de noms et prénoms dont la consonance - i.e. l'origine perçue qu'elle induit pour le recruteur - soit aussi clairement que possible (i) française, (ii) maghrébine ou (iii) étrangère avec une origine ethnique qui reste inconnue pour la population des recruteurs. La construction de ces noms et prénoms s'appuie sur une enquête préalable, au cours de laquelle une liste d'identités est présentée à un répondant à qui il est demandé d'identifier l'origine de la personne portant ce nom si celle-ci lui paraît certaine; et de laisser le champ vide dans le cas contraire. Nous souhaitons également pouvoir mettre ces différences de traitement liées aux origines en parallèle avec d'éventuelles différences liées au genre. A la question sur l'origine de l'identité s'ajoute donc une question sur le genre supposé à partir du prénom.

Pour être informative, l'enquête (décrite en annexe) doit être réalisée auprès d'individus représentatifs de la population des recruteurs qui recevront les candidatures envoyées dans le cadre de l'étude. Afin d'écarter tout risque de détection nous avons néanmoins choisi de ne pas interroger directement des recruteurs. Nous avons par conséquent sélectionné deux types de population : des employés chargés de la clientèle de divers établissements localisés dans la région parisienne, ainsi que des étudiants d'universités parisiennes. Sur la base de cette enquête préliminaire, nous retenons les noms et prénoms pour lesquels le pourcentage de réponses « correctes » (c'est à dire conformes à l'origine perçue pour laquelle le nom est utilisé comme variable de traitement) est le plus élevé dans chaque catégorie. 
Tableau 1 - Résultats de l'enquête: noms et prénoms à consonance française et maghrébine

\begin{tabular}{|c|c|c|c|c|c|}
\hline \multicolumn{3}{|c|}{ Noms-prénoms à consonance française (en \%) } & \multicolumn{3}{|c|}{ Noms-prénoms à consonance maghrébine (en \%) } \\
\hline & $\begin{array}{c}\text { Supposition } \\
\text { correcte }\end{array}$ & Genre perçu & & $\begin{array}{c}\text { Supposition } \\
\text { correcte }\end{array}$ & Genre perçu \\
\hline LECLERC Pascal & $99 \%$ & Masculin $97 \%$ & BENBALIT Rachid & $94 \%$ & Masculin $96 \%$ \\
\hline RIVIERE Benoît & $97 \%$ & Masculin 98\% & MOKRAOUI Yassine & $94 \%$ & Masculin $80 \%$ \\
\hline ROUSSET Sandrine & $97 \%$ & Féminin $\quad 98 \%$ & BENOUNIS Samira & $92 \%$ & Féminin $99 \%$ \\
\hline DUFOUR Jeanne & $96 \%$ & Féminin $93 \%$ & DERBAL Rachida & $88 \%$ & Féminin $98 \%$ \\
\hline
\end{tabular}

Lecture: 99\% des enquêtés perçoivent le nom LECLERC Pascal comme étant à consonance française et $97 \%$ le perçoivent masculin.

Source: enquête réalisée auprès des 300 individus.

Le Tableau 1 présente les résultats de l'enquête pour les deux identités à consonance française (partie gauche du tableau) et maghrébine (partie droite) qui arrivent en tête en termes de taux de réponses correctes. La partie supérieure du tableau présente ces résultats pour les identités masculines, la partie inférieure pour les identités féminines. Pour ces deux catégories d'origine, la perception qu'ont les enquêtés de la consonance et du genre des noms et prénoms proposés est sans équivoque et en parfait accord avec nos attentes. La quasitotalité des enquêtés affirme que les quatre noms de la partie gauche sont clairement d'origine française tandis que les quatre noms de la partie droite sont d'origine maghrébine.

En ce qui concerne le genre, une légère ambigüité apparaît à l'égard de $M O K R A O U I$ Yassine. En revanche, le genre des sept autres identités est presque unanimement reconnu par les enquêtés. Par conséquent, les identités masculine et féminine d'origine française retenues dans l'étude sont respectivement LECLERC Pascal et ROUSSET Sandrine. Quant aux noms d'origine maghrébine, nous retenons BENBALIT Rachid pour le candidat masculin et BENOUNIS Samira pour la candidate féminine. Par ailleurs, ces résultats conduisent à retenir les mêmes noms d'origine française et maghrébine de genre masculin que ceux de l'étude menée par Duguet et al. (2010). 
Tableau 2 - Résultats de l'enquête : noms et prénoms à consonance étrangère

\begin{tabular}{|c|c|c|c|c|c|c|c|c|}
\hline & \multicolumn{2}{|c|}{ Suppositions } & \multicolumn{6}{|c|}{ Autre origine majoritaire supposée } \\
\hline & Inconnu & Genre perçu & 1ère orig & & 2 nde origi & & 3ème orig & \\
\hline ALDEGI Jatrix & $83 \%$ & Masc. $73 \%$ & Pays de l'est & $5 \%$ & Europe du sud & $3 \%$ & Maghrébin & $3 \%$ \\
\hline KOCH Maynir & $65 \%$ & Masc. $53 \%$ & Maghrébin & $10 \%$ & Pays de l'est & $7 \%$ & Allemagne & $6 \%$ \\
\hline HADAV Alissa & $70 \%$ & Fém. $\quad 83 \%$ & Maghrébin & $10 \%$ & Pays de l'est & $7 \%$ & Israélien & $6 \%$ \\
\hline RAZEL Yuna & $55 \%$ & Fém. $\quad 76 \%$ & Maghrébin & $9 \%$ & Israélien & $6 \%$ & Pays de l'est & $6 \%$ \\
\hline
\end{tabular}

Lecture: $83 \%$ des enquêtés perçoivent le nom ALDEGI Jatrix comme étant d'origine étrangère mais inconnue et $73 \%$ estiment qu'il est masculin. Par ailleurs, $5 \%$ des enquêtés estiment que ce nom est originaire des pays de l'Est.

Source: enquête réalisée auprès des 300 individus.

Le Tableau 2 présente les résultats de l'enquête vis à vis des noms et prénoms qui recueillent les taux d'ignorance (i.e. champ vide, point d'interrogation, NSP) les plus élevés. Pour les deux noms qui seront retenus dans l'étude, ALDEGI Jatrix et HADAV Alissa, l'origine apparaît comme inconnue pour plus de deux enquêtés sur trois. Une différence importante apparaît entre ces deux candidats : les résultats sont beaucoup plus convaincants pour la candidature masculine que pour la candidature féminine. Il conviendra par conséquent d'interpréter avec précaution la discrimination observée à l'encontre de cette dernière candidature, principalement destinée à évaluer l'interaction entre les discriminations ethniques et les discriminations de genre. Les résultats sont beaucoup plus tranchés et satisfaisants au regard de cette dernière dimension. En dépit du manque de familiarité observé avec les identités proposées, le genre est «correctement» identifié par près de quatre répondants sur cinq. Il est important de noter pour la validité de l'étude que ces noms ne sont jamais identifiés par les répondants comme des noms d'origine française. Enfin, les origines déclarées par les répondants qui souhaitent se prononcer sont très variées tant en termes géographiques qu'en termes de stéréotypes physiques (Europe du sud, Maghreb, MoyenOrient). Il est ainsi hautement improbable que la discrimination éventuelle à l'égard de ces candidatures reflète une défiance à l'égard d'une origine particulière sur laquelle se concentreraient ces croyances résiduelles. Par conséquent, ces résultats permettent de valider l'interprétation selon laquelle ces noms fournissent une variable de traitement qui conduit les recruteurs à considérer ces candidats comme étant d'une origine (i) étrangère et (ii) inconnue.

\section{... des signaux dans les candidatures (variables de traitement II)...}

Les études récentes d'Oreopolous (2012) et Dechie et Oreopolous (2012) comptent parmi les investigations empiriques les plus complètes des sources de discrimination à l'embauche dues aux origines. L'un des résultats importants de ces études est que les employeurs déclarent utiliser l'origine déduite du nom du candidat pour en inférer son degré 
de maitrise du langage. Les résultats empiriques sont, eux, beaucoup plus contrastés puisque la mention explicite d'une activité témoignant d'une bonne maitrise du langage bénéficie plus aux candidats autochtones (anglo-saxons en l'occurrence) qu'aux candidatures à consonance étrangère.

Afin de tester cette source de discrimination statistique sur le marché du travail français, nous incluons une variable de traitement supplémentaire qui consiste à indiquer explicitement le niveau de maitrise de langue française du candidat à travers l'appartenance à un groupe de lecture, la participation à des concours de scrabble et de mots croisés ou encore une expérience dans le domaine de l'enseignement à travers des activités de tutorat et de soutien scolaire à domicile en français. Cette variable est intégrée aux réponses à une offre d'emploi sur deux. Lorsqu'elles le sont, ces indications sont ainsi ajoutées à l'ensemble des six candidatures envoyées à un même employeur.

Notre volonté d'étudier les discriminations liées au genre, d'autre part, nous conduit à dupliquer l'ensemble des candidatures avec des prénoms féminins. Or, l'identification du genre associé à l'identité de ces candidatures est fondamentale pour la validité des résultats. Ceci est d'autant plus vrai pour l'analyse de l'interaction entre les discriminations de genre et les discriminations liées aux origines si, comme on peut le penser, la capacité d'un employeur à identifier le genre lié à un prénom est d'autant plus faible que l'origine lui est peu familière. Les résultats de l'enquête constituent une première garantie à cet égard. Afin d'améliorer encore la qualité des perceptions, nous indiquons clairement sur les CV et lettres de motivation des différents candidats une information implicite faisant référence à leur genre. Par exemple, nous ajoutons volontairement des accords féminins (Forte d'une expérience, je suis motivée, etc.) ou utilisons le sigle « Mme » et «M. » dans l'en-tête du CV.

\section{... des caractéristiques du recruteur (variables de contrôle)...}

Parmi les caractéristiques de l'employeur dont nous disposons dans les offres d'emploi collectées (décrites ci-dessous), deux seront plus particulièrement utilisées dans l'analyse des résultats. Nous exploiterons, d'une part, la localisation géographique de l'entreprise et, d'autre part, les informations disponibles sur l'identité perceptible en termes de genre du (probable) recruteur.

L'une des conséquences importantes des comportements de nature discriminatoire est de renforcer et entretenir la ségrégation spatiale - i.e. la concentration des populations issues de l'immigration dans des zones géographiques circonscrites. Les résultats disponibles quant à la situation française de ce point de vue ne permettent pas de trancher clairement cette question. Du Parquet et al. (2011) montrent par exemple que la distance domicile-travail est sans effet sur la discrimination à l'embauche en Île-de-France - allant à l'encontre de l'hypothèse d'un renforcement de la ségrégation - tandis que Duguet et al (2012) observent un effet non négligeable (quoique concentré sur les candidatures féminines) de la composition 
sociale de la commune de résidence. Afin de nourrir ce débat, nous souhaitons croiser l'inégalité de traitement éventuelle que subissent les candidats avec la localisation géographique de l'employeur. Nous synthétiserons cette information sous la forme d'une variable indicatrice des localités situées à Paris par opposition à celles situées en banlieue afin de distinguer la localisation des employeurs qui reçoivent nos candidatures.

D'autre part, nous souhaitons enrichir notre analyse du rôle de l'homéophilie dans la discrimination en prenant en compte la proximité entre les caractéristiques du candidat et celles du recruteur. Or, la quasi-totalité des offres d'emploi que nous recueillons fournissent les coordonnées de la personne à contacter pour pouvoir postuler à l'offre. Ainsi, pour chaque offre d'emploi à laquelle les candidats fictifs ont répondu, le nom, le prénom et l'adresse mail de l'intermédiaire sont observés. Nous utiliserons ces informations pour classer les recruteurs en fonction du genre indiqué par leur nom. La pertinence de cette variable repose sur l'hypothèse que cet intermédiaire est bien le recruteur chargé de trier les candidatures. Bien que cette présomption soit renforcée par le fait que $72 \%$ des réponses que nous avons reçues émanent bien du contact apparaissant dans l'annonce, cette mesure reste une variable de contrôle bruitée puisqu'il reste envisageable que la personne à contacter ne dispose d'aucun pouvoir décisionnaire et soit uniquement chargée de la communication avec les postulants. En raison des biais qu'introduit cette propriété dans la modélisation, nous n'intégrerons cette variable qu'en complément d'analyses plus robustes.

\section{... ou de caractéristiques observables des postulants}

Afin de répondre au maximum d'offres d'emploi et ainsi maximiser les taux de réponses, il est souhaitable de se restreindre à des secteurs pour lesquels le marché de l'emploi est très actif et peu affecté par les fluctuations de l'activité économique. Pour cette raison, nous nous concentrons sur les professions liées à la comptabilité : comptable, assistant(e) et aide comptable. ${ }^{6}$

Nous répondons à chaque offre d'emploi collectée par l'envoi de six candidatures chacune portant l'une des identités décrites précédemment, soit un couple de candidatures homme/femme dont les noms et prénoms sont à consonance française, à consonance maghrébine et à consonance étrangère mais sans référence à un groupe démographique particulier.

\footnotetext{
6 D'après l'enquête «trajectoire et origines » de l'INED (publiée en 2010 et conduite en 2007-2008), les immigrés de seconde génération sont sur-représentés dans la catégorie des employés, des ouvriers et des professions intermédiaires. Les professions auxquelles correspondent les offres d'emploi retenues dans cette étude se situent dans ces catégories sociales (aide et secrétaire comptable correspond à la CSP des employés et comptable correspond à celle des professions intermédiaires). Enfin, les métiers de la comptabilité sont fortement féminisés, avec une proportion de femmes évaluée en 2009 à $58 \%$ (source: enquête emploi de l'Association Française des Banques).
} 
Dans la mesure où chaque employeur reçoit six candidatures de notre part, il est bien évidemment indispensable de différencier le contenu des CV correspondants afin d'éviter la détection de l'étude. Dans le même temps, les candidatures doivent être construites de manière à ce que les variables de traitement constituent les seules différences susceptibles d'influencer de manière systématique le succès relatif de nos différents envois. En conséquence, nous organisons une rotation systématique des identités sur chacun des six CV. Cette randomisation introduit un bruit dans le taux de succès associé à chaque catégorie (genre/origine) de candidat. Afin de limiter l'ampleur de ce bruit, nous construisons des candidatures aussi similaires entre elles que possible. Ainsi, les candidat(e)s sont célibataires et de nationalité française, sont âgés de 23 ans, habitent des quartiers socio-économiquement équivalents, sont dotés d'un Brevet de Technicien Supérieur (BTS) et ont une expérience professionnelle de 18 à 22 mois.

Plus précisément, les candidats sont nés en 1988 et n'ont jamais redoublé. Leur nationalité n'est jamais retranscrite explicitement sur le CV. L'adresse des candidats est réelle et située dans la moitié sud de Paris dans les $13^{\text {ème }}, 14^{\text {ème }}$ et $15^{\text {ème }}$ arrondissements. Les candidats sont dotés d'un BTS «Comptabilité et Gestion » obtenu en 2009. Cette formation est la plus largement demandée pour les emplois d'aide comptable et de comptable. Ainsi, le panel d'offres d'emploi auquel nous pouvons répondre est très important, et cette caractéristique assure l'attractivité de nos candidatures. Les candidats sont également dotés d'un baccalauréat Sciences et Technologies de la Gestion (STG) obtenu en 2007, dont certains avec mention. Cette filière a été choisie par souci de crédibilité, dans la mesure où $55 \%$ des élèves qui accèdent au BTS possèdent le baccalauréat STG.

Les candidats n'ont pas d'emploi au moment où ils postulent afin de signaler leur disponibilité immédiate. Ils possèdent une expérience professionnelle tirée de trois ou parfois deux emplois. Le premier emploi est toujours plus court, ce qui correspond au cas classique d'une période de stage effectuée après le BTS d'une durée comprise entre 2 et 6 mois. Les différentes expériences professionnelles et tâches associées qui sont reportées sur les CV sont véritables, en ceci qu'elles ont été tirées de CV réels apparaissant sur le site de pôle emploi. Seule la période d'activité associée à chaque emploi a été modifiée. Les intitulés des missions, de l'entreprise et de sa localité sont bien réels, mais ont été ré-appariés entre eux afin d'éviter une trop grande similarité avec des candidatures existantes. Enfin, les candidats ont travaillé à des postes en lien direct avec la comptabilité (aide comptable, assistant(e) comptable ou comptable) dans des entreprises différentes, toutes localisées dans la région Île-de-France.

Outre les différences de contenu, nous introduisons également des différences de forme entre les $\mathrm{CV}$, en termes de police d'écriture, de taille de cette police et de la mise en page des documents envoyés pour chacune des offres. Nous associons à chaque CV une lettre de motivation subissant le même type de modifications de forme. Si elles diffèrent dans leur formulation et par l'ordre dans lequel apparaissent les différents éléments, ces lettres sont similaires sur le fond, et s'inspirent en particulier d'exemples directement tirés de sites internet spécialisés. 


\section{De l'envoi des candidatures...}

Les offres d'emploi sont recueillies quotidiennement sur le site de Pôle Emploi. Cette source est complétée par des sites Internet spécialisés dans les offres d'emploi (APEC.fr ; cadreemploi.fr). Nous répondons à toutes les offres d'emploi respectant les critères suivants : emploi à temps complet ou partiel, $\mathrm{CDI} / \mathrm{CDD}$ et localisé dans la région Ile-de-France. Aucune candidature spontanée n'a été envoyée et les candidatures parviennent aux recruteurs le jour de la parution des offres. La grande majorité des CV est envoyée par courrier électronique. Nous avons donc créé au préalable six adresses mail et attribué six numéros de téléphone portable aux candidats. En réponse aux candidatures postées, les employeurs qui le souhaitent peuvent contacter le candidat par e-mail ou téléphone en y laissant un message vocal. Les annonces de messagerie sont standards et automatisées.

Chaque employeur reçoit six CV, dont l'expédition est décalée dans le temps. L'ordre d'envoi des candidatures a été randomisé de sorte qu'une identité particulière ne soit pas systématiquement envoyée en première. De plus, un signal attestant d'une «bonne » maîtrise de la langue française est ajouté sur les CV des différents candidats pour la moitié des offres d'emploi sollicitées.

La principale mesure d'intérêt est le nombre de réponses positives reçues par chacune des identités de candidat. Une réponse de la part d'un recruteur est considérée comme positive si le candidat est directement convié à un entretien ou si le recruteur se manifeste pour obtenir plus de renseignements sur sa situation présente ou ses qualifications. En revanche, une réponse négative est attribuée à une candidature si le recruteur la rejette formellement ou simplement s'il n'y répond pas. Comme pour toute étude de ce type, cette mesure présente l'inconvénient de ne pas renseigner directement sur le succès de la procédure d'embauche. Elle constitue une mesure fidèle de la discrimination si la probabilité d'embauche conditionnellement à une convocation à un entretien est la même quels que soient le genre et l'origine du candidat; et une mesure biaisée si cette probabilité est corrélée avec l'identité du candidat. Il faut cependant noter que nos résultats sous-estiment l'ampleur de la discrimination dès lors que, comme c'est probable, l'identité joue sur cette probabilité dans la même direction que sur les chances de convocation à un entretien. Cette question n'a à notre connaissance jamais été explorée dans la littérature empirique.

\section{... aux résultats de l'évaluation}

Les résultats préliminaires présentés ci-dessous résultent d'une première série d'envois qui s'est déroulée de septembre à Novembre 2011. Ils proviennent des réponses à 300 offres d'emploi, ce qui correspond à l'envoi de $1800 \mathrm{CV}$. Les données obtenues sont analysées selon trois dimensions : l'ampleur de la discrimination d'origine et le rôle de l'homéophilie, 
Tableau 3 - Taux de réponses favorables par candidature

\begin{tabular}{|c|c|c|c|c|c|}
\hline \multicolumn{2}{|c|}{ Consonance française } & \multicolumn{2}{|c|}{ Consonance maghrébine } & \multicolumn{2}{|c|}{ Consonance étrangère } \\
\hline Candidat & Réponses & Candidat & Réponses & Candidat & Réponses \\
\hline LECLERC Pascal & $48 \quad 16,0 \%$ & BENBALIT Rachid & $217,0 \%$ & ALDEGI Jatrix & $279,0 \%$ \\
\hline ROUSSET Sandrine & $62 \quad 20,7 \%$ & BENOUNIS Samira & $42 \quad 14,0 \%$ & HADAV Alissa & $44 \quad 14,7 \%$ \\
\hline \multirow{2}{*}{ Ensemble } & \multirow{2}{*}{$110 \quad 18,3 \%$} & Ensemble & $63 \quad 10,5 \%$ & Ensemble & $71 \quad 11,8 \%$ \\
\hline & & \multicolumn{4}{|c|}{ Non français : 134 réponses ; $11,2 \%$ de réponses positives } \\
\hline
\end{tabular}

Lecture: le candidat masculin d'origine française Leclerc Pascal a reçu 48 réponses positives suite à sa candidature aux 300 offres d'emploi. Le taux de réponses positives de ce candidat équivaut donc à $16 \%$.

Champ: réponses aux 300 offres d'emploi dans la région IDF.

l'importance des discriminations liées au genre et l'influence de la discrimination statistique liée à l'inclusion d'un signal de maitrise du langage.

Les principaux résultats découlent de la comparaison du nombre de réponses positives (et le taux de rappel correspondant) qu'ont reçu chacun de nos candidats en fonction à la fois de l'origine perçue de leur nom et de son genre (cf. Tableau 3). Pour la candidature de référence (un homme dont le nom est à consonance française) le taux de rappel est de l'ordre de 1 sur 6 - un candidat dont le nom est à consonance française doit répondre à six offres d'emploi pour décrocher un entretien d'embauche. Cette proportion concorde avec celle que retrouvent la plupart des études consacrées à ce sujet, ce qui tend à valider le protocole de l'étude en termes de contenu des candidatures comme de secteur d'activité.

La comparaison globale des taux de réponses positives fait très clairement apparaître l'existence d'une inégalité de traitement entre les candidatures de différents types. Les envois qui ont reçu le plus de réponses favorables sont ceux portant un nom à consonance française $(18,3 \%$ en moyenne contre $11,2 \%)$. Selon ces premiers résultats, une candidature d'origine française reçoit donc en moyenne presque une fois et demie plus de réponses positives qu'une candidature qui ne l'est pas à CV égal. La décomposition des taux de rappel selon les origines indique que l'identification de l'origine précise du candidat n'a pas d'influence sur son succès : les taux de rappel des candidats d'origine étrangère mais inconnue $(11,8 \%)$ et maghrébine $(10,5 \%)$ sont tout à fait comparables. En effet, si nos tests statistiques (cf. Tableau 4) indiquent que la différence de traitement entre les candidatures d'origine française et celles d'origines maghrébine et étrangère est significative à $1 \%$, ils nous informent aussi que les deux candidatures d'origine non française sont traitées de manière égale. 
Tableau 4 - Tests statistiques de Student selon l'origine et le genre

Discrimination d'origine conditionnellement au genre masculin

\begin{tabular}{|c|c|c|}
\hline $\mathrm{H} 0:$ français = maghrébin & $\mathrm{H} 0:$ français = étranger & $\mathrm{H} 0:$ maghrébin $=$ étranger \\
$\mathrm{H} 1:$ français $\geq$ maghrébin & $\mathrm{H} 1:$ français $\geq$ étranger & $\mathrm{H} 1:$ maghrébin $\neq$ étranger \\
$\mathrm{t}$-test $=4,58^{* * *}$ & $\mathrm{t}$-test $=3,62 * * *$ & $\mathrm{t}$-test $=-1,42$ \\
\hline
\end{tabular}

Discrimination d'origine conditionnellement au genre féminin

\begin{tabular}{|c|c|c|}
\hline $\mathrm{H} 0:$ français = maghrébin & $\mathrm{H} 0:$ français = étranger & $\mathrm{H} 0:$ maghrébin $=$ étranger \\
$\mathrm{H} 1:$ français $\geq$ maghrébin & $\mathrm{H} 1:$ français $\geq$ étranger & $\mathrm{H} 1:$ maghrébin $\neq$ étranger \\
$\mathrm{t}$-test $=3,29 * * *$ & $\mathrm{t}$-test $=3,04 * * *$ & $\mathrm{t}$-test $=-0,43$ \\
\hline
\end{tabular}

Discrimination de genre conditionnellement à l'origine

\begin{tabular}{|c|c|c|}
\hline Origine française & Origine maghrébine & Origine étrangère \\
\hline $\mathrm{H} 0:$ masculin $=$ féminin & $\mathrm{H} 0:$ masculin $=$ féminin & $\mathrm{H} 0:$ masculin $=$ féminin \\
$\mathrm{H} 1:$ masculin $\neq$ féminin & $\mathrm{H} 1:$ masculin $\neq$ féminin & $\mathrm{H} 1:$ masculin $\neq$ féminin \\
$\mathrm{t}$-test $=-2,07 * *$ & $\mathrm{t}$-test $=-3,86 * * *$ & $\mathrm{t}$-test $=-3,00 * * *$ \\
\hline
\end{tabular}

Lecture: la statistique de test de comparaison des taux de réponses des candidats masculins d'origine française et maghrébine (issus de la table 3.1) est égale à 4,58, indiquant une différence de traitement statistiquement significative au seuil de $1 \%$. De manière générale, ***, ** et * indiquent que les différences de traitement observées entre deux candidatures sont significatives aux seuils respectifs de 1\%, 5\% et $10 \%$.

Champ: les tests sont implémentés sur la base des résultats issus de la table 3.

Une inégalité de traitement apparaît en fonction du genre entre les candidats de même origine. Les différences de taux de succès sont systématiquement favorables aux candidatures féminines, quelle que soit l'origine perçue du nom. : le taux de succès des candidatures à consonance française passe de $20,7 \%$ pour les candidates à $16,0 \%$ pour leur équivalent masculin, de $14,0 \%$ à $7,0 \%$ pour les noms à consonance maghrébine et $14,7 \%$ à $9,0 \%$ pour les noms à consonance étrangère dont l'origine est inconnue. Les différences de traitement observées ici sont d'ailleurs toutes significatives au seuil de $1 \%$ (cf. Tableau 4 Discrimination de genre conditionnellement à l'origine). 
Tableau 5 - L'effet du signal de maîtrise du langage sur les taux de rappels

\begin{tabular}{|c|c|c|c|c|c|c|c|c|}
\hline \multicolumn{3}{|c|}{ Consonance française } & \multicolumn{3}{|c|}{ Consonance maghrébine } & \multicolumn{3}{|c|}{ Consonance étrangère } \\
\hline Candidat & Neutre & Signal & Candidat & Neutre & Signal & Candidat & Neutre & Signal \\
\hline LECLERC Pascal & $14,7 \%$ & $17,3 \%$ & BENBALIT Rachid & $4,0 \%$ & $10,0 \%$ & ALDEGI Jatrix & $8,0 \%$ & $10,0 \%$ \\
\hline ROUSSET Sandrine & $21,3 \%$ & $20,0 \%$ & BENOUNIS Samira & $10,0 \%$ & $18,0 \%$ & HADAV Alissa & $10,7 \%$ & $18,7 \%$ \\
\hline Ensemble & $18,0 \%$ & $18,7 \%$ & Ensemble & $7,0 \%$ & $14,0 \%$ & Ensemble & $9,3 \%$ & $14,3 \%$ \\
\hline
\end{tabular}

Lecture: le taux de réponses positives du candidat masculin d'origine maghrébine Benbalit Rachid est de 10,0\% lorsque le signal attestant d'une "bonne" maîtrise de la langue est ajouté au CV, alors que ce taux n'est que de 4\% lorsque le signal n'est pas ajouté.

Champ: réponses aux 300 offres d'emploi dans la région IDF.

Toutefois, l'effet de l'origine décrit plus haut est indifférencié selon que l'on compare les taux de rappel entre hommes ou entre femmes. Parmi les candidats masculins, le candidat français est convoqué à un entretien dans $16,0 \%$ des cas contre $7,0 \%$ si le nom porté sur la candidature est à consonance maghrébine et 9,0\% s'il est à consonance étrangère dont l'origine est inconnue. Les tests de comparaison de moyennes entre ces taux de rappel indiquent que les différences de traitement observées entre le candidat d'origine française et les candidats d'origines maghrébine et étrangère sont significatives au seuil de $1 \%$. Ces différences de traitement sont très légèrement atténuées parmi les candidatures féminines mais restent toujours significatives au seuil de $1 \%$.

En résumé, les résultats issus de cette série d'envois indiquent une inégalité de traitement significative à l'encontre de toutes les candidatures qui apparaissent comme issues de l'immigration. Pour les trois profils de candidats, on remarque que les candidatures féminines apparaissent privilégiées en comparaison de leurs équivalents masculins. Enfin, conformément à l'hypothèse selon laquelle la discrimination répond à un sentiment d'homéophilie ethnique, les candidats dont le nom n'est pas à consonance française sont également discriminés dans le processus d'embauche.

\section{La présence d'un signal de maîtrise du langage améliore sensiblement le taux de convocation des candidats quelle que soit leur origine}

Comme indiqué plus haut, 150 des 300 groupes de candidatures envoyées comportent une indication explicite destinée à attester d'une bonne maitrise de la langue française. L'objectif de cette différenciation des envois est de mesurer l'une des causes de la dimension statistique de la discrimination, liée aux compétences linguistiques. 

Tableau 6 - Tests binomiaux selon l'origine et le genre lorsque les candidatures
incorporent le signal de langage

\begin{tabular}{|c|c|c|}
\hline \hline \multicolumn{3}{c}{ Discrimination d'origine conditionnellement au genre masculin } \\
\hline français $(14)$ - maghrébin (3) & français $(12)$ - étranger $(1)$ & maghrébin $(4)$ - étranger (4) \\
\hline $\mathrm{H} 0: 14 /(14+3)=1 / 2$ & $\mathrm{H} 0: 12 /(12+1)=1 / 2$ & $\mathrm{H} 0: 4 /(4+4)=1 / 2$ \\
$\mathrm{H} 1: 14 /(14+3) \geq 1 / 2$ & $\mathrm{H} 1: 12 /(12+1) \geq 1 / 2$ & $\mathrm{H} 1: 4 /(4+4) \geq 1 / 2$ \\
Test binomial $: \mathrm{p}$-value $=0,00^{* * *}$ & Test binomial $: \mathrm{p}$-value $=0,00^{* * *}$ & Test binomial : p-value $=0,64$ \\
\hline
\end{tabular}

Discrimination d'origine conditionnellement au genre féminin

\begin{tabular}{|c|c|c|}
\hline français $(8)$ - maghrébin (5) & français $(7)$ - étranger $(5)$ & maghrébin (5) - étranger (6) \\
\hline $\mathrm{H} 0: 8 /(8+5)=1 / 2$ & $\mathrm{H} 0: 7 /(7+5)=1 / 2$ & $\mathrm{H} 0: 6 /(5+6)=1 / 2$ \\
$\mathrm{H} 1: 8 /(8+5) \geq 1 / 2$ & $\mathrm{H} 1: 7 /(7+5) \geq 1 / 2$ & $\mathrm{H} 1: 6 /(5+6) \geq 1 / 2$ \\
Test binomial $: \mathrm{p}$-value $=0,29$ & Test binomial $: \mathrm{p}$-value $=0,38$ & Test binomial $: \mathrm{p}$-value $=0,50$ \\
\hline
\end{tabular}

Discrimination de genre conditionnellement à l'origine

\begin{tabular}{|c|c|c|}
\hline $\begin{array}{c}\text { Origine française } \\
\text { masculin }(9)-\text { féminin }(13)\end{array}$ & $\begin{array}{c}\text { Origine maghrébine } \\
\text { masculin }(2)-\text { féminin }(14)\end{array}$ & $\begin{array}{c}\text { Origine étrangère } \\
\text { masculin }(2)-\text { féminin }(15)\end{array}$ \\
\hline $\mathrm{H} 0: 13 /(9+13)=1 / 2$ & $\mathrm{H} 0: 14 /(2+14)=1 / 2$ & $\mathrm{H} 0: 15 /(2+15)=1 / 2$ \\
$\mathrm{H} 1: 13 /(9+13) \geq 1 / 2$ & $\mathrm{H} 1: 14 /(2+14) \geq 1 / 2$ & $\mathrm{H} 1: 15 /(2+15) \geq 1 / 2$ \\
Test binomial $: \mathrm{p}$-value $=0,26$ & Test binomial $: \mathrm{p}$-value $=0,00^{* * *}$ & Test binomial $: \mathrm{p}$-value $=0,00^{* * *}$ \\
\hline
\end{tabular}

Lecture: lorsque les candidatures incluent le signal de langage, le candidat masculin dont le nom est à consonance française a été préféré au candidat d'origine maghrébine 14 fois, alors que ce dernier n'a été préféré qu'à 3 reprises. Le test binomial associé aux écarts de traitement entre les candidatures masculines d'origine française et maghrébine indique une probabilité critique (ou p-value) nulle, impliquant une forte préférence pour ces premières relativement aux secondes. De manière générale, $* * *, * *$ et $*$ indiquent que les différences de traitement observées entre deux candidatures sont significatives aux seuils respectifs de 1\%, 5\% et 10\%.

Champ: les tests sont implémentés sur la base des résultats issus de la table 5 dans le cas où les candidatures incorporent le signal de langage.

Le tableau 5 désagrège les résultats présentés dans le tableau 3 en fonction de la présence ou non d'un signal de maitrise du langage. Pour toutes les identités considérées, la mention d'une activité qui témoigne d'une bonne maitrise du français améliore toujours les taux de rappel observés. Notons d'abord que toutes les conclusions discutées jusqu'ici sur la base de l'ensemble des candidatures sortent renforcées si l'on se concentre uniquement sur les candidatures exemptes de signal: ni les discriminations de genre ni les discriminations d'origine ne sont donc imputables à la présence de cette variable dans une partie des envois.

Le tableau 6 présente les statistiques de tests de comparaison des taux de réponses positives obtenues par les candidats en fonction de la présence du signal de maîtrise de langue. Comme pour les comparaisons présentées dans le Tableau 4, nous nous concentrons sur les neufs couples de candidats formés par les comparaisons deux à deux entre origines et entre genres. Dans le cas de l'effet du signal, ces comparaisons conduisent à réduire de moitié la taille de l'échantillon. Il convient donc d'utiliser des tests plus performants à distance finie que les tests de Student (Aeberhardt et al., 2009). Nous utilisons des tests binomiaux, fondés 


\section{Encadré 1 : Construction du test binomial}

Les tests dits paramétriques, tels que le test de Student, reposent sur des hypothèses de distribution asymptotique (loi normale en l'occurrence) qui ne sont valides que pour de grands échantillons. Lorsque les échantillons sont de petite taille (dans notre cas, lorsqu'il s'agit de tester l'effet de la maîtrise de la langue française sur la discrimination à l'embauche, où le nombre d'observations est de 150) les résultats issus de tests non paramétriques, qui ne reposent sur aucune hypothèse de distribution, sont donc plus fiables. Cet encadré décrit les modalités de mise en œuvre du test non paramétrique utilisé dans le texte.

Nous souhaitons tester la significativité des écarts de traitement entre les candidatures de même genre mais d'origine différente, ainsi que les différences entre les candidats masculins et féminins de même origine. Le test binomial est donc utilisé pour tester les écarts à l'intérieur des neufs couples de candidatures définis dans le Tableau 4. Pour mettre en œuvre ce test, nous nous restreignons aux offres pour lesquelles une candidature particulière a été privilégiée à une autre (traitement inégalitaire). Nous retenons donc pour chacune des deux candidatures en question, le nombre de réponses positives obtenues lorsque celle-ci a été préférée à l'autre dans le processus de recrutement. Cette situation correspond à une inégalité de traitement puisque seule l'une des deux candidatures a été convoquée à l'entretien d'embauche. Ensuite, nous calculons la proportion de réponses positives «privilégiées» obtenues par la candidature potentiellement non discriminée, soit celle qui a été la plus privilégiée dans le processus de recrutement. Cette proportion s'obtient en divisant le nombre de réponses favorables obtenues par cette candidature lorsqu'elle a été préférée par la somme des réponses obtenues par chacune d'entre elles dans le cas d'un traitement inégalitaire. Une proportion égale à $1 / 2$ indique une égalité de traitement entre les deux candidats. A l'inverse, plus cette proportion tend vers 1 , et plus le traitement de ces deux candidatures est inégalitaire.

Le test est construit de sorte que sous l'hypothèse nulle, les candidats sont traités sur un pied d'égalité, c'est-à-dire lorsque la proportion de réponses privilégiées équivaut à $1 / 2$. L'hypothèse alternative de ce test correspond à la situation où cette proportion est supérieure à $1 / 2$. Le traitement des deux candidatures est donc inégalitaire et en défaveur de la candidature potentiellement discriminée si le test conduit à rejeter l'hypothèse nulle.

sur la proportion relative du nombre de réponses positives obtenues par l'un des deux candidats lorsque celui-ci a été préféré à son homologue (ce nombre est à droite de l'origine du candidat et entre parenthèse dans le Tableau 6). L'hypothèse nulle d'égalité de cette proportion à $1 / 2$ correspond à un traitement identique des deux candidatures. Une proportion significativement supérieure à cette valeur seuil indique, en revanche, qu'elles font l'objet d'un traitement inégal. La construction précise du test binomial est détaillée dans l'encadré 1. 
Bien qu'il soit négligeable pour la candidature féminine d'origine française, l'inclusion du signal de langage dans les candidatures engendre une hausse du taux de rappel pour son homologue masculin. Ces deux inflexions de sens opposés sont conjointement suffisantes à absorber les différences de succès liées au genre : dès lors que les candidatures font mention d'une expérience qui atteste d'une bonne maitrise de la langue française, les hommes et les femmes dont le nom est à consonnance française sont traités sur un plan d'égalité. D'ailleurs, la très faible probabilité critique issue du test binomial nous indique qu'aucune différence de traitement significative n'existe entre ces deux candidatures lorsque le signal est inclus.

Tous genres confondus, la présence d'un signal de maitrise du langage améliore sensiblement le taux de convocation des autres candidats, et ce dans des proportions tout à fait comparables entre les différentes origines : le taux de rappel passe de 7,0\% à 14,0\% pour les candidatures à consonance maghrébine, de $9,3 \%$ à $14,3 \%$ pour les noms à consonance étrangère dont l'origine est inconnue. Mais pour ces deux catégories de candidats, une asymétrie oppose les candidatures masculines aux candidatures féminines.

Bien que l'effet du signal améliore notablement et dans des proportions importantes les chances de succès des candidatures féminines, il est légèrement plus faible pour les candidatures masculines. Compte tenu de la légère inflexion à la baisse subie par les candidatures féminines à consonance française, ce premier effet est sufisant à éliminer toute discrimination d'origine entre les candidatures féminines. D'ailleurs, les tests binomiaux de traitement égalitaire indiquent qu'aucune inégalité de traitement n'est significative entre les candidates d'origine française et les candidates d'origines maghrébine et étrangère.

Sur la base de ces résultats, la discrimination de genre qui s'exerce à l'encontre du candidat français semble gouvernée par des considérations statistiques, fondées sur de meilleures compétences attendues en matière linguistique. Cette asymétrie dans les compétences perçues par les employeurs se retrouve dans les mêmes termes en matière de discrimination d'origine : une fois les compétences liguinstiques explicitées, les femmes de toutes origines sont traitées sur un plan d'égalité.

\section{Les discriminations selon les caractéristiques du recruteur seraient plutôt le fait de son genre (féminin) et de sa localisation}

Afin d'analyser l'effet des caractéristiques du recruteur sur le degré de discrimination et évaluer la robustesse des résultats discutés jusqu'ici, nous concluons cette étude par une décomposition de nos résultats en fonction des caractéristiques de l'emploi pour lequel les candidatures sont envoyées. Trois dimensions sont utilisées : le type d'emploi (assistant(e) comptable, secrétaire comptable ou comptable), la localisation géographique de l'entreprise (Paris ou sa banlieue) et enfin le genre du correspondant à qui sont envoyées les candidatures. 
Tableau 7 - Ratio de discrimination et caractéristiques de l'emploi sollicité

\begin{tabular}{|l|ccc|cc|cc|}
\cline { 2 - 8 } \multicolumn{1}{c|}{} & \multicolumn{3}{c|}{ Type d'emploi } & Genre du recruteur & \multicolumn{2}{c|}{$\begin{array}{c}\text { Localisation de } \\
\text { l'entreprise }\end{array}$} \\
\cline { 2 - 9 } & $\begin{array}{c}\text { Assistant(e) } \\
(123)\end{array}$ & Secrétaire & Comptable & Homme & Femme & Paris & Banlieue \\
LECLERC Pascal & 1,00 & 2,08 & 1,23 & 1,00 & 0,55 & 1,00 & 1,34 \\
BENBALIT Rachid & 3,43 & 4,16 & 2,01 & 6,51 & 1,02 & 3,33 & 2,50 \\
ALDEGI Jatrix & 2,19 & 4,15 & 1,70 & 3,25 & 0,82 & 2,50 & 2,06 \\
ROUSSET Sandrine & 0,92 & 1,13 & 0,88 & 0,93 & 0,36 & 0,91 & 0,90 \\
BENOUNIS Samira & 1,41 & 1,04 & 1,70 & 1,18 & 0,63 & 1,54 & 1,29 \\
HADAV Alissa & 1,34 & 1,56 & 1,23 & 1,30 & 0,51 & 1,33 & 1,25 \\
\hline
\end{tabular}

Lecture: 123 des 300 offres d'emploi auxquelles nous avons répondu s'adressaient à des assistant(e)s comptables, 64 à des secrétaires comptables et 113 à des comptables. Pour obtenir le même nombre de convocations à un entretien d'embauche pour un emploi d'assistant comptable, par exemple, le candidat dont le nom est à consonance maghrébine doit envoyer 3,43 candidatures contre une pour le candidat dont le nom est à consonance française.

Champ: réponses aux 300 offres d'emploi dans la région IDF.

La première conclusion qui émerge de cette décomposition (Tableau 7) est qu'aucune des discriminations que nous avons observées n'est spécifique ni à un type d'emploi particulier, ni à une localisation, ni à une catégorie de recruteurs : quelle que soit la dimension considérée, les inégalités de traitement demeurent défavorables aux candidatures d'origine maghrébine et étrangère pour le groupe des hommes comme pour celui des femmes. De même, conditionnellement à l'origine, les candidatures féminines apparaissent toujours privilégiées en comparaison de leurs homologues masculins.

La décomposition des taux de rappel selon le type d'emploi indique toutefois que les écarts de succès entre les différentes identités sont fortement atténués pour les emplois de comptable. L'avantage de genre dont bénéficient les candidatures féminines atteint son plus haut niveau sur les postes de secrétaire. La décomposition des taux de réponses positives selon les caractéristiques du recruteur fait apparaître deux résultats saillants. D'une part, si les candidatures féminines sont privilégiées par tous les types de recuteurs, cet avantage de genre tend à s'amplifier lorsque les candidatures sont traitées par un recruteur de genre féminin. Pour les noms à consonance française, cet effet est même seul responsable des différences de genre observées : alors que la différence de traitement observée entre les deux candidatures françaises est non significative lorsque le recruteur est un homme, elle le devient au seuil de $1 \%$ lorsque ce dernier est une femme. Il semble donc que les recruteurs de genre "féminin" aient tendance à favoriser leurs homologues de même sexe pour tenter de réduire les inégalités de traitement dans l'accès à l'emploi qui jouent généralement en leur défaveur. D'autre part, les résultats indiquent que le degré de discrimination ethnique tend à se réduire lorsque l'entreprise sollicitée est localisée en banlieue parisienne. Il semble donc que la discrimination à l'embauche liée à l'origine favorise la ségrégation spatiale. 
Les résultats préliminaires issus de l'évaluation par correspondance décrite dans cet article confirment l'existence d'une forte discrimination à l'embauche de candidats clairement identifiés comme issus de l'immigration, mais sans identification précise de leur origine. Cette discrimination est d'ampleur identique à celle que subissent les candidats issus d'une immigration d'origine maghrébine. Ces résultats suggèrent que l'homéophilie ethnique (qui consiste à réserver un traitement inégalitaire aux individus sur la base de leur appartenance ou non au groupe ethnique majoritaire) constitue un moteur important des comportements discriminatoires sur le marché du travail. Si ces résultats sont cohérents avec l'existence d'une relation entre homéophilie ethnique et discrimination, ils ne permettent pas d'en mesurer l'intensité. Une question importante à cet égard serait de savoir dans quelle mesure la distance ethnique (perçue) affecte l'ampleur de la discrimination, et quels en sont les déterminants.

Ensuite, la discrimination observée dans cette étude est très sensible à l'inclusion dans les candidatures d'une expérience faisant explicitement référence à des compétences linguistiques. Le degré de discrimination envers les candidats d'origine maghrébine ou étrangère tend à se réduire nettement lorsqu'un signal de maîtrise du langage est ajouté sur le $\mathrm{CV}$. Cet effet est cependant fortement asymétrique en fonction du genre : il est suffisant à éliminer toute discrimination d'origine entre les candidatures féminines, mais ne fait qu'atténuer légèrement la discrimination qui affecte les candidatures masculines. Ce résultat vient confirmer l'idée que les attentes de la part des employeurs sont assez différentes en fonction du genre du candidat. En l'absence de signal de maitrise du langage, les femmes françaises sont assez nettement favorisées ; mais cet avantage sur leurs homologues issues de l'immigration disparaît lorsque les compétences linguistiques sont explicitement mentionnées. Ces signaux permettent donc d'interpréter plus finement la dimension statistique de la discrimination. L'information qu'ils véhiculent ne se limite cependant pas au contenu de l'expérience dont ils témoignent: ils attestent également d'une vie associative active, d'une volonté d'implication au delà de la sphère professionnelle etc.... Si l'interprétation en termes de maitrise de la langue nous paraît donc la plus cohérente avec le contenu effectif du signal ajouté aux candidatures, elle n'en restera pas moins spéculative tant que son effet ne sera pas comparé à celui d'autres signaux, de même nature mais indépendant des compétences linguistiques. Ces craintes paraissent cependant limitées au regard des résultats de Bougard et al. (2011), qui n'observent aucun effet des activités de bénévolat sur la probabilité de convocation à un entretien d'embauche.

Enfin, et plus généralement, ces résultats restent, comme toute évaluation, conditionnels au périmètre de l'étude, en termes de type d'emploi, de secteur d'activité ou encore de zone géographique. L'inférence des effets observés à l'ensemble du marché du 
travail repose sur l'hypothèse que le périmètre choisi ne présente pas de spécificités en termes de propension à discriminer (liées par exemple aux préférences des recruteurs, ou encore au degré de concurrence dans le recrutement). Sous ces différentes réserves, les résultats présentés ici apportent des éléments de réponse nouveaux quant aux instruments susceptibles de contenir la discrimination.

D'abord, l'existence d'une discrimination envers les minorités visibles est fréquemment invoquée pour valider l'adoption de plus en plus fréquente de programmes de discrimination positive (affirmative action aux Etats-Unis), destinés à compenser temporairement les inégalités de traitement entre individus de différentes origines. Pour des raisons pratiques évidentes, les mesures de discrimination positive sont appliquées à des minorités ethniques clairement identifiées. Dans la mesure où nos résultats suggèrent l'existence d'une défiance généralisée à l'égard de tout individu n'appartenant pas au groupe ethnique dominant, ce type de programme ne peut-être que partiellement efficace. En effet, les mesures de favoritisme n'élimineront que la discrimination envers les minorités ethniques concernées. Toute intervention fondée sur l'identification de groupes ethniques particuliers est donc condamnée à laisser inchangée la situation des individus qui, bien qu'issus de l'immigration, échappent à ces catégorisations.

L'une des originalités de notre étude est ensuite d'identifier le degré de maîtrise du langage comme une dimension déterminante du raisonnement statistique qui sous-tend le comportement des employeurs. La mention explicite d'une expérience extra-professionnelle liée à l'usage du langage réduit en effet la discrimination d'origine quel que soit le genre du candidat - même si cet effet est fortement atténué pour les candidatures masculines. Ce mécanisme peut être exploité assez aisément dans le cadre d'une intervention publique destinée à lutter contre les discriminations, en instaurant par exemple un système de labellisation du niveau de maîtrise du langage, qui pourrait prendre la forme d'un examen écrit et/ou oral et dont la mise en œuvre pourrait être confiée aux organismes de gestion des demandeurs d'emploi. Pour avoir un effet sur les compétences perçues des candidats, il faut que ce label soit de nature à être mentionné sur les candidatures (d'où la nécessité d'une procédure nationale, plus lisible pour les employeurs), et que cette mention soit vérifiable pour éviter qu'il fasse l'objet de manipulations : l'organisme gestionnaire (Pôle emploi par exemple) pourrait à cette fin être chargé de mettre cette information à disposition des employeurs qui en font la demande. Nous résultats suggèrent en outre qu'il est souhaitable que ce signal concerne tous les chercheurs d'emploi, y compris si leur identité ne les conduit pas à être perçus comme issus de l'immigration. Enfin, ce label doit être crédible du point de vue des compétences qu'il vise à signaler, et devrait donc être accompagné de programmes de formation au français pour les candidats qui échouent à en atteindre le niveau. Par extrapolation, les résultats présentés dans cet article laissent penser que ce type d'intervention participerait à aligner les croyances des employeurs avec les compétences effectives des candidats, et donc à atténuer les discriminations. Il reste que seule une expérimentation sociale de ce type de mesure peut permettre d'en évaluer l'efficacité réelle. 


\section{Annexe 1 : Construction de la liste de noms et prénoms des candidats}

Les noms et prénoms utilisés dans l'étude sont extraits d'une liste de 32 couples nom-prénom préalablement choisis par nos soins. Cette liste a été construite en s'inspirant des travaux existants et en nous appuyant sur des sources publiques d'information sur les noms et prénoms les plus courants par origine et par genre.

Nous demandons à chaque individu de renseigner, s'il n'a pas de doute, l'origine et le genre supposés des 32 identités décrites par les noms et prénoms présentés. L'étude est réalisée auprès d'un panel de 300 individus, dont la moitié sont employés de cinq établissements différents de la région parisienne. Quatre de ces cinq établissements appartiennent au secteur public, le dernier est un établissement privé. La seconde moitié des enquêtés est exclusivement composée d'étudiants de premier cycle universitaire. Notre souci dans l'échantillonnage des répondants a été d'utiliser une souspopulation qui, à la fois, soit représentative de la population des recruteurs qui recevront nos candidatures; mais qui simultanément, ne se trouvent pas eux mêmes en position de recruteur dans leur établissement, afin d'éviter tout risque d'identification de l'expérience. L'enquête est présentée dans la figure ci-dessous.

Les résultats complets, et le contenu exhaustif, de l'enquête sont disponibles sur demande auprès des auteurs.

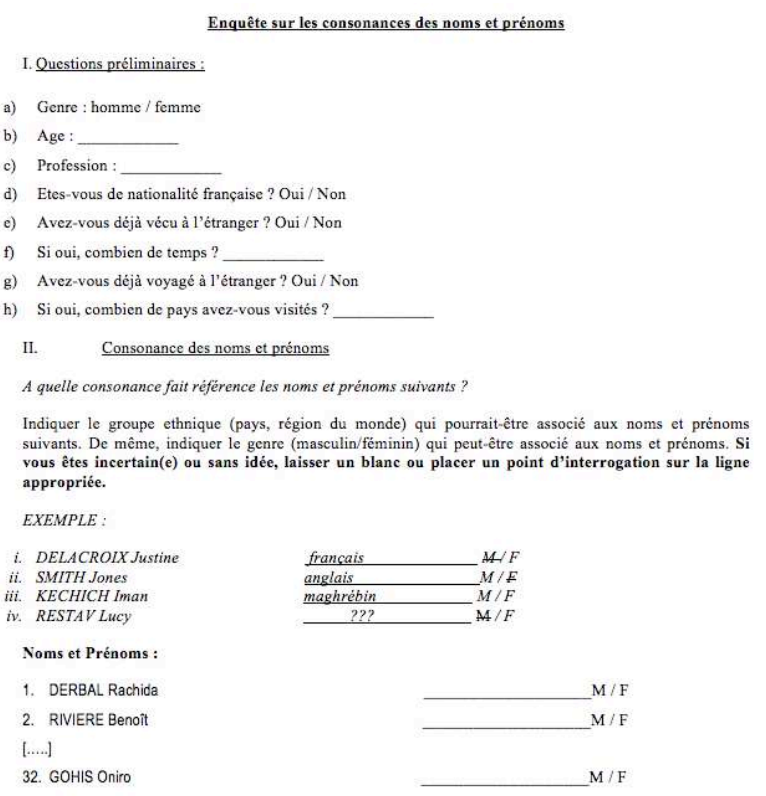

\section{Références bibliographiques}

Adida C.L., Laitin D.D. et Valfort M.A. (2010), « Identifying barriers to Muslim integration in France », Proceedings of the National Academy of Sciences, vol 107, ${ }^{\circ} 52$, pp. 1-7.

Aeberhardt R., Fougère D., Pouget J. et Rathelot R. (2010), « Wages and Employment of FrenchWorkers with African Origin », Journal of Population Economics, vol 23, n 3, pp. 881-905. 
Aeberhardt R., Fougère D. et Rathelot R. (2009), « Discrimination à l'embauche : comment exploiter les procédures de testing ? », Document de travail DESE, INSEE, n G2009-13.

Alesina A. et Ferrara E.L. (2005), « Ethnic Diversity and Economic Performance », Journal of Economic Literature, vol 43, $\mathrm{n}^{\circ} 3$, pp.762-800.

Becker G. S. (1971), « The Economics of Discrimination », University of Chicago Press, Chicago (IL).

Berson C. (2012), « Does Competition Induce Hiring Equity ? », CES Working Paper, $\mathrm{n}^{\circ}$ 12019.

Berson C. (2009), « Private vs. Public Sector: Discrimination against Second-Generation Immigrants in France », CES Working Paper n09059.

Bertrand M. et Mullainathan S. (2005), « Are Emily and Greg More Employable than Lakisha and Jamal? A Field Experiment on Labor Market Discrimination », American Economic Review, vol. 94, n 4, pp. 991-1013.

Bougard J., Brodaty T., Emond C., L'Horty Y., du Parquet L. et Petit P. (2011), « Les effets du bénévolat sur l'accès à l'emploi : une expérience contrôlée sur les jeunes qualifiés d'Île-de-France », Document de travail du CEE, n 147.

Bovenkerk F., Kilborne B., Raveau F. et Smith D. (1979), " Comparative Aspects of Research on Discrimination Against Non-White Citizens in Great Britain, France and the Netherlands ", Problems in International Comparative Research in the Social Sciences, pp. 105-122.

Cédiey E. et Foroni F. (2007), «Les discriminations à raison de « l'origine » dans les embauches en France : une enquête nationale par tests de discrimination selon la méthode du Bureau International du Travail », Rapport pour le Bureau International du Travail.

Crenshaw K. (1991), " Mapping the Margins: Intersectionality, Identity Politics, and Violence against Women of Color Stanford Law Review », Stanford Law Review, vol 43, n 6, pp. 1241-1299.

Currarini S., Jackson M. O. et Pin P., (2009), « An Economic Model of Friendship: Homophily, Minorities, and Segregation », Econometrica, vol 77, n 4, pp. 1003-1045.

Dechie D. et Oreopoulos P. (2012), « Why do some employers prefer to interview Matthew but not Samir? New evidence from Toronto, Montreal and Vancouver », CLSRN Working Paper.

Duguet E., Leandri N., L'Horty Y. et Petit P. (2010), « Are Young French Jobseekers of Ethnic Immigrant Origin Discriminated Against? A Controlled Experiment in the Paris Area », Annals of Economics and Statistics, vol 99-100, pp. 187-216. 
Duguet E. et Petit P. (2005), " Hiring Discrimination in the French Financial Sector: An Econometric Analysis on Field Experiment Data », Annals of Economics and Statistics, vol 78, pp. 79-102.

Duguet E., L'Horty Y., Du Parquet L., Petit P. et Sari F. (2012) « Les effets du lieu de résidence sur l'accès à l'emploi : une expérience contrôlée sur des jeunes qualifiés en Île-deFrance », Economie et Statistique, à paraître.

Du Parquet, L., Duguet, E., L'Horty, Y. Petit, P. et Sari, F. (2011), « Être mobile pour trouver un emploi ? Les enseignements d'une expérimentation en région parisienne», Rapport de recherché $T E P P, \mathrm{n}^{\circ} 3$.

Fershtman C. et Gneezy U. (2001), " Discrimination in a Segmented Society: An Experimental Approach », Quarterly Journal of Economics, vol 116, n 1, pp. 351-77.

Firth M. (1980), " Racial discrimination in the British labor market », Industrial and Labor Relations Review, vol 34, n², pp. 265-272.

Firth M. (1982), « Sex discrimination in job opportunities for women », Sex Roles, vol 8, ${ }^{\circ}$ 8, pp. 891-901.

Habyarimana J., Humphreys M., Posner D. N. et Weinstein J. M. (2009), « Coethnicity: Diversity and the Dilemmas of Collective Action », Russell Sage Press, New York (NJ).

Heckman J. J. (1998), « Detecting Discrimination », Journal of Economic Perspectives, vol $12, \mathrm{n}^{\circ} 2$, pp. 101-116.

Jacquemet N. et Yannelis C. (2011), «Indiscriminate Discrimination: A Correspondence Test for Ethnic Homophily in the Chicago Labor Market », Labor Economics, Vol. 19 (6), pp. 824-832

Jowell R. et Prescott-Clark P. (1970), « Racial Discrimination and White-Collar Workers Workers in Britain », Race, vol 11, n 4, pp. 397-417.

Miguel E. et Gugerty M. K. (2005), « Ethnic diversity, social sanctions, and public goods in Kenya », Journal of Public Economics, vol 89, n 11-12, pp. 2325-2368.

Lang K. et Lehman J.Y.K. (2012), « Racial Discrimination in the Labor Market: Theory and Empirics », Journal of Economic Literature, vol.50 n4, pp. 959-1006.

Oreopoulos P. (2011), « Why Do Skilled Immigrants Struggle in the Labor Market? A Field Experiment with Thirteen Thousand Resumes », American Economic Journal: Economic Policy, vol 3, n 4, pp. 148-171. 
Ottaviano G.I.P. et Peri G. (2006), «The economic value of cultural diversity: evidence from US cities », Journal of Economic Geography, vol 6, n 6, pp. 9-44.

Du Parquet L., Duguet E., L'Horty Y., Petit P. et Sari, F. (2010), « Être mobile pour trouver un emploi ? Les enseignements d'une expérimentation en région parisienne », Document de travail de l'EPEE, $\mathrm{n}^{\circ} 140$.

Petit P. (2004), « Discrimination à l'embauche. Une étude d'audit par couples dans le secteur financier », Revue économique, Presses de Sciences-Po, vol. 55, n 3, pp. 611-621.

Phelps E. S. (1972), « The Statistical Theory of Racism and Sexism », American Economic Review, vol 62, n 4, pp. 659-661.

Putnam R. D. (2007), « E Pluribus Unum: Diversity and Community in the Twenty-first Century », Scandinavian Political Studies, vol 30, n² 2, pp. 137-174.

Riach P.A. et Rich J. (2002), " Field Experiments of Discrimination in the Market Place », Economic Journal, vol 112, n483, pp. 480-518. 